\title{
Minimization of Sink Mark Defects in Injection Molding Process Optimization of a Composite Drive Shaft Used in Automobiles
}

\author{
Anwarullah, SP. Arun Kumar
}

\begin{abstract}
The motive for this paper is to show an included technique for improving the excellent attributes of the infusion fashioned component (Honda Upper Part) being fabricated at assembling industry (Pakistan), wherein the dismissal price for Honda higher element became entrance ramp due to sink marks abandons. The proposed coordinated method grasps the concept of Design of Experiments (Taguchi Approach) and Response floor structure philosophy for infusion forming procedure enhancement. The Taguchi Method (TM) was utilized to brief rundown the elements that effectsly affect the sink tests in infusion shaped components. Moreover, the development method of Response Surface Methodology (RSM) changed into used for the trial research to comfy a forecast model that may be applied to decorate infusion shaping process as some distance as firstrate sink marks decrease. The outcome suggests that the sink marks lower

Anticipated by way of the coordination of the Taguchi Method and RSM in truth dwindled from zero.0088 (Taguchi's final results) all the way down to $0.0080 \mathrm{~mm}$. The genuine effects find that the becoming a member of of the Taguchi Method and RSM should safely improve the great. Essentially with those examinations, we attempted to open the imaginative and prescient of manufacturers and architects with regard to using included RSM/Taguchi approach using instantly cum connection relapse version with selected parametric/ranges placing for the dimensional exactness of the infusion shaped part, and in this way contributing closer to progress in procedure's unwavering high-quality. The incorporated method with relapse model for enhancement would not simply supply hypothetical well worth to the writing and makers/fashioners but can likewise be applied to diverse assembling organisations for high-quality elements technology.
\end{abstract}

Watchwords: Injection Molding Process Optimization, Sink Marks, Design of Experiments, Taguchi Approach, Response Surface Methodology

\section{INTRODUCTION}

Over the maximum latest 30 years, plastics have come to be the maximum most important constructing material for most gadgets. We look into the most famous varieties of plastics, and how they may be prepared.

Plastics has become the essential drivers of improvements and new object improvement. Employments of plastics are interwoven in all elements of our contemporary manner of life, and are becoming important; objects constituted of plastics run from subtle restorative inserts to expendable circle of relatives executes. Canny and innovative utilization of plastics have spread out new roads inside the polymerhardware region; from natural light transmitting diodes to electro-optical and bioelectrical supplements, from

Revised Manuscript Received on September 14, 2019.

Md. Anwarullah, Principal\& Professor, Siddhartha Institute of Technology \&Sciences, Narapally, Hyderabad, Telangana, India.

SP. Arun Kumar, Professor, Siddhartha Institute of Technology \&Sciences, Narapally, Hyderabad, Telangana, India. minimum attempt plastics chips to adaptable solar powered cells. A portion of the plastics can direct even strength and discharge mild, prompting development of ingenious programs. Extraordinary repute being used of plastics can be visible in automobiles and in a extensive assortment of modern programs.

All plastics are polymers; those polymers are additionally remoted into essential kinds: thermoplastics and thermo sets. Thermoplastics dissolve when warmed - so that they can be liquefied and re-fashioned over and over. Thermosets solidify when they are warmed, on every occasion warmed further, they will separate artificially and lose their residences. Some thermo units have residences essentially similar to elastic, and are utilized as manufactured elastic they're taken care of as elastomers. Here are a few run of the mill plastics and their employments:

\section{METHODOLOGY}

General houses: more and more strong, harder, excessive, light.

Common uses: car components, improvement materials.

The maximum broadly identified techniques for managing plastics to produce plastic parts are like techniques we've learnt for metals and glass. These comprise Extrusion, Injection shaping, Blow forming, Casting, and so forth. Among these, maybe infusion shaping is the most huge for neighborhood industry - practically all assembling cutting-edge sample of item development is in the direction of lighter and littler gadgets yet it's miles relied upon to carry awesome and viable preparations. Exquisite and respectable searching flimsy walled infusion formed nooks and housings are in style. Concentrated consideration at the issues of shape and assembling tactics can be visible from the growth of studies exercises. Considerable quantities of examinations had been accounted for on infusion shaping procedure enhancement, when all is stated in accomplished, and plenty of on the sink marks, specifically. The examinations did over the maximum recent few a long time relied on hypothetical, FE circulation exercise fashions and right down to earth take a look at preliminaries. This phase introduces a factor via point survey of looks into within the ongoing past on sink marks

An early and hanging examination approximately shrinkage, warpage and sink marks was directed by way of Chang and Tsaur (1995). In light of the rheological homes of polymers, an incorporated speculation and PC software changed into created on this investigation for copy of such

Published By:

Blue Eyes Intelligence Engineering

\& Sciences Publication 


\section{MINIMIZATION OF SINK MARK DEFECTS IN INJECTION MOLDING PROCESS OPTIMIZATION OF A COMPOSITE DRIVE SHAFT USED IN AUTOMOBILES}

behavior. Half breed restrained component and limited assessment strategies have been applied for mimicking the infusion shaping filling, pressing, and cooling bureaucracy. A manipulate extent technique changed into carried out closer to each locating the liquefy the front role and moreover ascertaining the temperature and weight profile at any second during the filling system. An adjusted Tait condition offers an outline of the weight extent-temperature courting of crystalline polymers. The Malkin's strength version became applied to painting the conduct of polymer crystallization. The movement actuated and heat triggered leftover burdens utilized because the underlying conditions in the robust mechanics examination have been gotten with the direct thermoviscoelastic model. The relocations, consisting of the thickness. Heading of part, which couldn't be determined by means of the normal bowing minute method, were settled by making use of the numerical sturdy mechanics examination with the three dimensional constrained element techniques. The hypothetical consequences were additionally related utilising commercial enterprise FE circulation reenactment programming. In spite of the truth that the coordinated program had the choice to reenact shrinkage and warpage on shaped gadgets, the sink reproduction turned into not tested definitively

\section{APPROACH}

Test set-inside the mood for leading the pastime checks contains of the precise traditional plastics part shape, confined aspect version of the part, determination of a reasonable assessment of thermoplastics forming cloth, an infusion shaping machine and a form configuration subtleties.

\subsection{D Part version}

Three Dimensional (three-D) parametric part model is required because the fundamental info. The 3-d part version may be created from any CAD programming geared up for sending out alternatives for simulation.In this contextual evaluation, Honda Upper Part (object by means of Alsons Auto Pvt. Ltd.) become taken for tests. The Sink marks changed into set apart as one of the large imperfection for the maximum part took place in formed Honda higher part causing many assembling line dismissal. The created strategy became carried out to the Injection forming accommodations. Reenactment preliminaries taken in order that to test how Sinks Marks deformity adjustments as we exchange the parametric settings. The Honda higher component was planned making use of the CAD programming. It changed into meant to its size as in keeping with the component drawing measurement facts gave by using the corporation. The element and its CAD configuration are regarded in Figure-five. The improvement goal in the above investigation is the main factor to be lit up before beginning the examination. The goal of the investigation is to decide the association of parameters which prompts the exceptional part shape potential with most reduced Sink Marks. The definition of the objective prompts the distinguishing evidence of fundamental factors and the portrayal of system execution variable. The potential area for the trim parameters became characterized thinking procedure activity of the Honda Upper Part

about the data reachable in the writing, specialised data sheets, and assembling grasp's feeling.

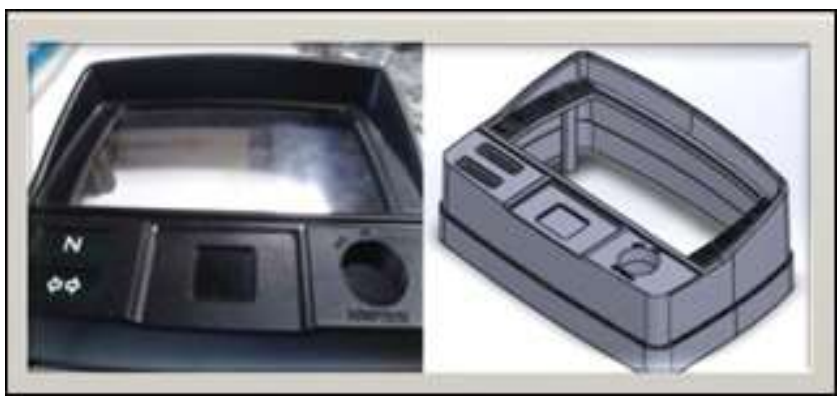

Figure 1:3D model3.2 Methods

\subsection{Taguchi method}

A DoE technique is useful to be unique potential that expands Taguchi Quality method parameter area are examined via analysis negligible number. Taguchi technique to handle excellent control applies to the complete manner of creating and assembling items from the begin the concept to collect / generation in PC meeting inserted condition. Taguchi method is the right utilization of systems and inspection assessments with the last aim of sturdy planning and best improvement. Taguchi accomplish this intention via making callous manner to yield sorts to be had in spite of the reality that the commotion in the method as illustrated by using the P-chart (Figure 2). The procedure is then said to have were given STOUT (Y.P Tidke et al 2014).

How to handle the boom in Taguchi, to the mixture is performed and the capacity parameters by using placing parametric procedure has attractions for producers and creators to run a progressive mechanical applications. Taguchi technique parametric shape has been used inside the past to prepare the parameters distinguish large and growing parametric settings. SH. Tang et al. (2006) has a bottom plate broke a bit at the bouncy, while doing ANOVA making use of symmetric cluster L9 Taguchi. Creator filtered out softening temperature, urgent time and urgent weight as vital additives even as occupying time is not crucial to the warpage of deformity. Feng, Chung et al. (2006) analyzed the diverse

\section{RESULTS AND DISCUSSION}

\subsection{FE version for recreational circulation}

In this examination MoldFlow this, programming is used to show the procedure of formation of the infusion. Side results from the reproductive circulate of extraordinarily excellent FE contribution to the program information. Information huge Info integrate affordable version to make FE work, getting ready essential information on deciding on and forming polymer material information. Information and different sorts of polymer cloth statistics that may be acquired from the dedication of fabric sheets.Using standard method restrained mid-plane component version created by using the go segment of a 3-d version with the aid of 1684 directly triangular shell additives. Everyday attitude 
percentage of the work used changed into 1,528. Works absolutely checked to throw an blunders associated work. Framework feeding and cooling channels are made depending on the plan form.

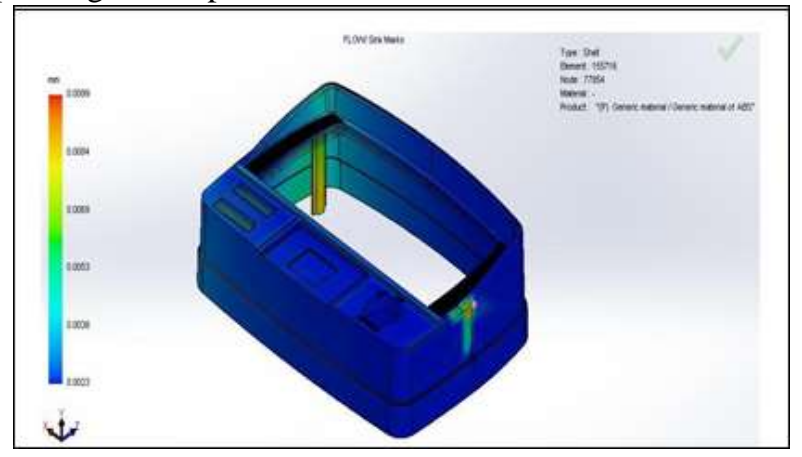

\section{CONCLUSION}

This paper shows a sturdy blend of the two instrument restore to progress the exploration of process improvement models. Plan techniques to avoid exploration enjoy standards and techniques of Trial-and-errors this is usually used for the improvement of this segment. The fee of the take a look at is predicted to achieve, the technique of a robust function, and increase throughput generation is also decreased. The following consequences were determined:

The effects show that downsizing as a ways as sink marks as a minimum derived from the technique included as 0.0080 , a figure superior to any sink mark receives upgraded with Taguchi approach as a stand alone.Three parameters without delay (Mold Temperature, Pressure Holding Time and occupied the time) and 01 communications ( (Mo * Ho) has a critical impact on the growth at least sink this path, the outcomes additionally showed that square things between parameters inconsequential impact on the sink mark

\section{REFERENCES}

1. Sanjay Lahoti, Prof M.D. Nadar, S. Kulkarni (2013) Optimization of Process Parameters plastic injection shaping the International Journal of Advanced Engineering Research and Studies E-ISSN2249-8974.

2. SH. Tang, Y.J. Tan, B.C. Swoop, S. Solomon, N. Ismail, R. Samin (2007). Utilization Taguchi strategy within the shape of infusion plan plastic to lessen warpage. Diary of Materials Processing Technologypp 418-426.

3. B. Ozcelik and Sonat I. (2009). Structural inspection bouncy and plastic shell forming tiny plastic infusion. Materials and 367-375 Designpp

4. Pak Aditya M. Darekar, Prof. T. S. Venkatesh, Dr T. Bhushan Patil, N. MrYazad Specialist (2015). Audit Aspects of Optimization for Plastic Injection Molding Process. IRACST - Engineering Science and Technology: An International Journal (ESTIJ), ISSN: 2250-3498 Vol.Five, No.1,

5. Feng Chung; Jeffrey Kuo; and Su, Te-Li. (2006), Optimization of Multiple Quality Characteristics for polyether ether ketone Injection Molding Process, Fiber and Polyers, vol.7, No.Four, pp 404-41

6. Yung-TsanJou, Wen-Tsann Lin, Wei-Cheng lee1 and Tsu-Ming Yeh (2014): "Entering the Taguchi Method and Response Surface Methodology Optimization of Injection olding foProceParameter" Applied Mathematics and Information Sciences An International Journal-Appl. Mathematics. Inf. Sci. 8, No. 3, 1277-1285 